ISSN 1420-3049

www.mdpi.org/molecules

Article

\title{
Synthesis of an ent-Halimanolide from ent-Halimic Acid
}

\author{
Isidro S. Marcos ${ }^{1, *}$, Almudena Conde ${ }^{1}$, Rosalina F. Moro ${ }^{1}$, Pilar Basabe ${ }^{1}$, David Díez ${ }^{1}$, \\ Faustino Mollinedo ${ }^{2,3}$ and Julio G. Urones ${ }^{1}$
}

${ }^{1}$ Departamento de Química Orgánica, Universidad de Salamanca, Plaza de los Caídos 1-5, 37008 Salamanca, Spain

${ }^{2}$ Centro de Investigación del Cáncer, Instituto de Biología Molecular y Celular del Cáncer, Consejo Superior de Investigaciones Científicas-Universidad de Salamanca, Campus Miguel de Unamuno, E37007 Salamanca, Spain.

${ }^{3}$ APOINTECH, Campus Miguel de Unamuno, E-37007 Salamanca, Spain

* Author to whom correspondence should be addressed; E-mail:ismarcos@usal.es

Received: 28 April 2008; in revised form: 7 May 2008 / Accepted: 7 May 2008 / Published: 9 May 2008

\begin{abstract}
An efficient synthesis of ent-halimanolide 2 (15,16-epoxy-12-oxo-ent-halima5(10),13(16),14-trien-18,2 $\beta$-olide), from ent-halimic acid has been achieved, corroborating the structure of the natural compound and establishing its absolute configuration.
\end{abstract}

Keywords: Ent-halimanolides, ent-halimic acid, diterpenoids, Cladogynos orientalis.

\section{Introduction}

Euphorbiaceae plants are a rich source of bioactive substances [1-2] and certain genera of this family have attracted much interest, since they contain a group of antitumor compounds [3]. Cladogynos orientalis Zipp. ex Span. (syn. Adenochlaena siamensis Ridl.) (Euphorbiaceae), known in Thailand as "Chettaphangki," is the only member of the genus Cladogynos and the roots are used as a carminative in Thai folk medicine. Chettaphanin I [4-5] and II [6-7], are the main components from their roots of this plant and the first to be known. Recently, in addition to chettaphanin I and II, 
isolation from the root extract of a series of furan diterpenes 2-4 with ent-halimane skeletons has been described [8].

Figures 1. Structures of furo-ent-halimanes from Cladogynos orientalis and ent-halimic acid.

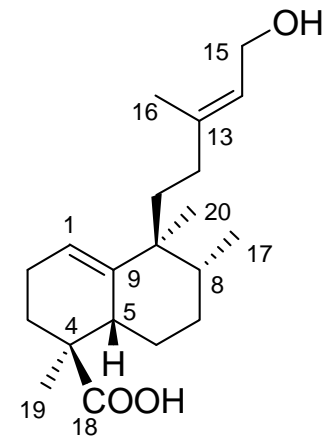

ent-halimic acid, 1<smiles>C[C@H]1CC[C@H](C)[C@]2(O)C(=CC(=O)C[C@]2(C)C(=O)O)[C@H]1CC(=O)c1ccoc1</smiles>

chettaphanin I<smiles>C[C@H]1CCC2=C3C(=C(c4ccoc4)C[C@@]3(C)C(=O)O)C(=O)C[C@@]2(C)C1</smiles>

chettaphanin II

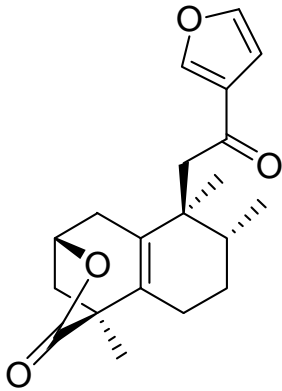

2

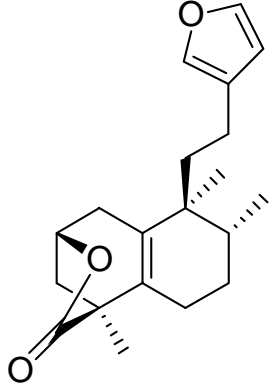

3<smiles>C[C@H]1CCC2=C(CCC[C@@]2(C)C(=O)O)[C@@H]1CCc1ccoc1</smiles>

4

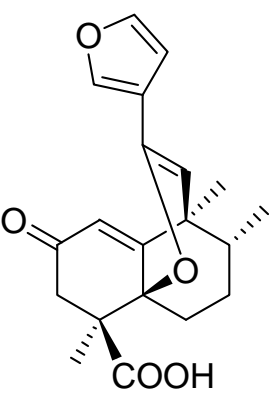

5

Our group has transformed ent-halimic acid 1, a diterpene of known structure and absolute configuration, into chettaphanin I and II, which confirmed their structure and absolute configuration $[4,6]$. In this paper, we report the synthesis of ent-halimanolide 2, in order to confirm the structure of the natural compound and do SAR studies. ent-Halimanolide is a furan diterpene like chettaphanin I and II, but in this case the carboxylic acid at C-18 has formed a $\gamma$-butanolide with the hydroxyl at C-2.

\section{Results and Discussion}

In order to synthesise compound $\mathbf{2}$, from ent-halimic acid $\mathbf{1}$ it is necessary to functionalize C-2 and C-12, add a furan group in the side chain, isomerize the double bond to the more stable tetrasubstituted position and to form the lactone ring.

Two synthetic routes have been explored for the synthesis of 2: Route A and Route B, which differ in the strategy followed for the preparation of the $\gamma$-lactone, before or after of the introduction of the furan ring.

In Route A (Scheme 1) three fundamental parts can be differentiated: elaboration of the adequate ent-halimic acid tetranorderivative $\mathbf{9} ; \gamma$-lactone formation as $\mathbf{1 2}$, necessary for the final introduction of the furan fragment and to prepare the functional groups required to achieve the natural product 2. 


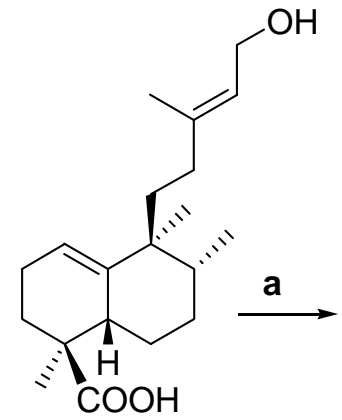

1

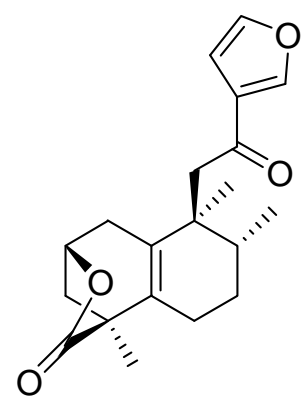

2

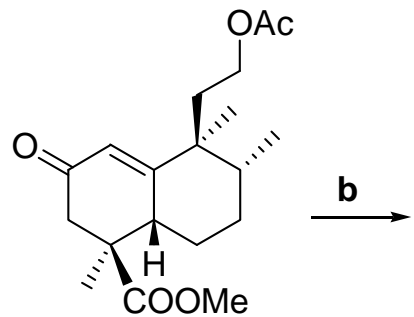

6<smiles>COC(=O)OCC[C@]1(C)CC2(CC[C@H]1C)OCCO2</smiles>

7<smiles>CC(=O)OCC[C@]1(C)CCC2=C(CC(=O)C[C@@]2(C)C(C)=O)[C@@H]1C</smiles>

8

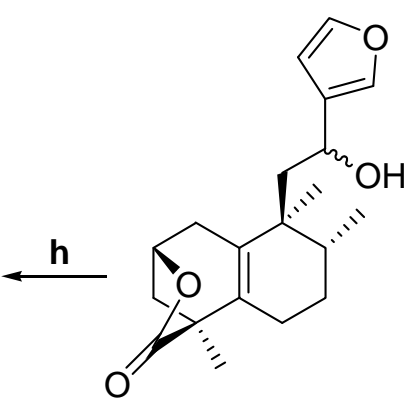

$1312 S$
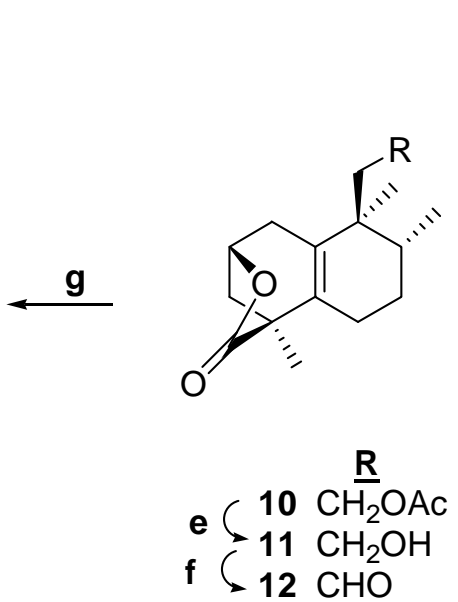

a) Ref. 3a; b) ethylene glycol, $p$-TsOH, $\mathrm{C}_{6} \mathrm{H}_{6}$, reflux, 8h (97\%); c) HCl 2M, EtOH, 3h (92\%); d) $\mathrm{NaBH}_{4}$, EtOH, rt, 3h (9: 47\%, 10: 49\%); e) $\mathrm{Na}_{2} \mathrm{CO}_{3}, \mathrm{MeOH}$, rt, 5 h (97\%); f) TPAP, NMO, DCM, rt, 30 min (96\%); g) 3-bromofuran, n-BuLi (1/1), -78 ${ }^{\circ} \mathrm{C}, 30 \min (13$ : 54\%, 14 : 36\%); h) TPAP, NMO, DCM, rt, 50 min (92\%).

The ent-halimic acid tetranorderivative $\mathbf{6}$ was obtained in excellent yield as described in the synthesis of chettaphanin I and II [4] [6]. Carbonyl protection of $\mathbf{6}$ with ethylene glycol in acid media gives the tetranorderivative 7 , that already contains the tetrasubstituted double bond in the required position. The carbonyl deprotection should be done very carefully (controlling the acid and time) and in this manner ketone 8 can be obtained, which by $\mathrm{NaBH}_{4}$ reduction gives a 1:1 mixture of hydroxyderivative $\mathbf{9}$ and $\gamma$-lactone $\mathbf{1 0}$.

The required aldehyde $\mathbf{1 2}$ was obtained by hydrolysis of $\mathbf{1 0}$ followed by TPAP [9] oxidation of the hydroxy derivative 11. Addition of 3-furyl lithium [10-12] to 12 gives a mixture of the hydroxy derivatives 13 and 14. The C-12 configuration in 13 and 14 was established by comparation of their physical properties with the ones of similar compounds [13-14]. Oxidation of the mixture of $\mathbf{1 3}$ and $\mathbf{1 4}$ with TPAP gives $2,[\alpha]_{\mathbf{D}}^{22}-101.4\left(\mathrm{c} 0.2, \mathrm{CHCl}_{3}\right.$ ), that was identical in all its physical properties to the natural compound 15,16-epoxy-12-oxo-ent-halima-5(10),13(16),14-trien-18,2 $\beta$-olide, $[\alpha]_{\mathbf{D}}^{22}-151.5$ (c 0.017, $\mathrm{CHCl}_{3}$ ), already described [8].

Route B (Scheme 2) involves first a new procedure for the synthesis of the key intermediate, the tetranorderivative 19 already used by our group in the synthesis of chetaphanin I and II, and secondly the transformation of this intermediate into the natural compound 2. Our new route for the synthesis of 
intermediate 19 gives a better global yield than one based on a Baeyer-Villiger reaction as a key step $[4,6]$, and can be done in a multigram scale.

\section{Scheme 2}<smiles>COC(=O)[C@]12CC[C@@H](C)[C@]1(CCC(C)=O)C1=CC(=O)C[C@@H](C)[C@]12C</smiles>

15

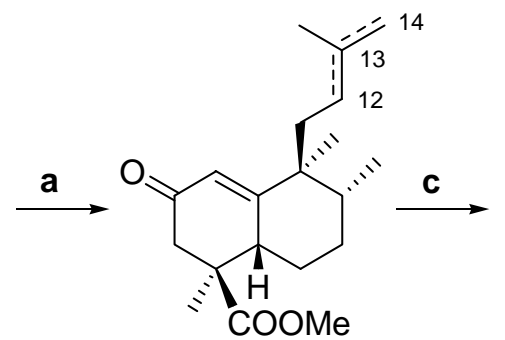

bC $\begin{array}{ll}16 & \Delta^{13(14)} \\ 17 & \Delta^{12}\end{array}$<smiles>CC(=O)O[C@]1(C)CC[C@H](C)[C@H](CC=C(C)C)C1=C1CC2(CC1C)OCCO2</smiles>

18<smiles>COC(=O)[C@]1(C)CC2=C(CC3(C2)OCCO3)[C@@](C=O)(CC=O)[C@@H]1C</smiles>

19 e

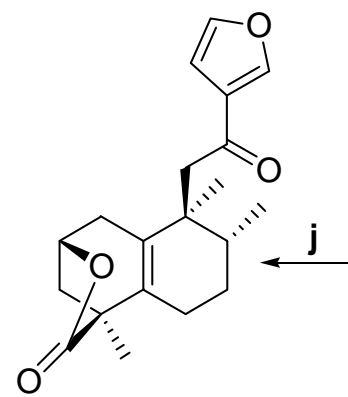

2

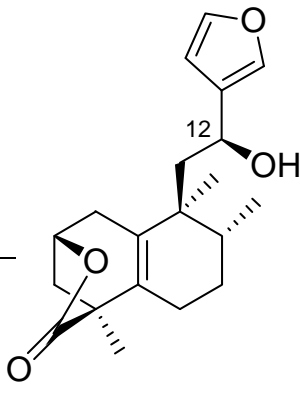

13
27

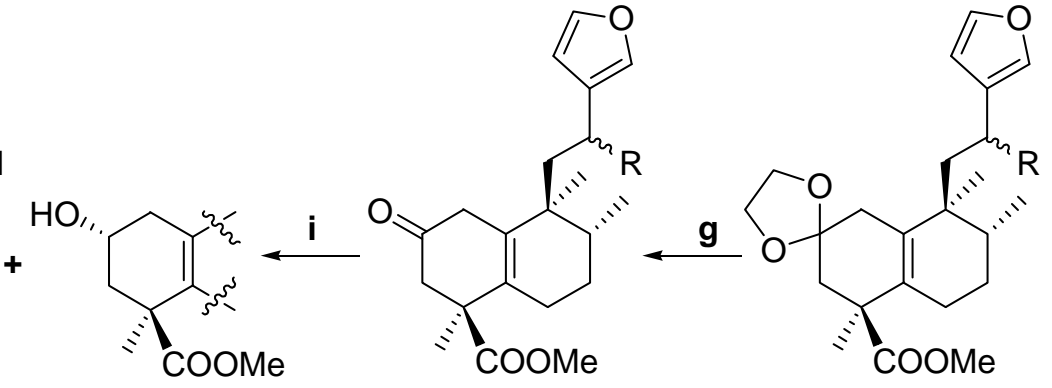

$\mathbf{R}$

a) $\mathrm{MePPh}_{3} \mathrm{Br}, \mathrm{NaHMDS}$, THF, $-78^{\circ} \mathrm{C}, 30 \mathrm{~min}$ (94\%); b) $p$-TsOH, $\mathrm{C}_{6} \mathrm{H}_{6}, 60^{\circ} \mathrm{C}$, 2h (96\%); c) $\left(\mathrm{CH}_{2} \mathrm{OH}\right)_{2}, p$-TsOH, $\mathrm{C}_{6} \mathrm{H}_{6}$, Dean Stark, 16 h, (97\%), d) 1.- OsO ${ }_{4}, \mathrm{NMO},{ }^{t} \mathrm{BuOH}$, THF, $\mathrm{H}_{2} \mathrm{O}$, rt, overnight; 2.- $\mathrm{Pb}(\mathrm{AcO})_{4}, \mathrm{C}_{6} \mathrm{H}_{6}$, rt, 39 min (96\%, two steps); e) 3-bromofuran, $n$-BuLi, THF, 30 min (20 : 54\%, 21 : 39\%); f) $\mathrm{Ac}_{2} \mathrm{O}$, pyridine 12 h (98\%); g) HCl-2M, EtOH, rt, 3 h (96\%); h) $\mathrm{Na}_{2} \mathrm{CO}_{3}, \mathrm{MeOH}, 2$ h, (96\%); i) $\mathrm{NaBH}_{4}$, EtOH, rt, 3h (13: 38\%, 27: 43\%); j) TPAP, NMO, DCM, rt, 50 min (92\%).

Starting from 15, previously obtained from ent-halimic acid, 1 [4], by chemoselective Wittig reaction [15-17], we obtained $\mathbf{1 6}$. Treatment of $\mathbf{1 6}$ under acidic conditions allows the isomerization of the side chain terminal double bond into the more stable trisusbstituted position in quantitative yield, to give compound 17. The protection of the carbonyl group gives the dioxolane 18, with concomitant isomerization of the double bond in the bicyclic system to the tetrasusbsituted position. Chemoselective oxidation of the side chain of $\mathbf{1 8}$ was achieved by treatment with $\mathrm{OsO}_{4}$ [18-21], followed by cleavage of the resulting diol with $\mathrm{Pb}(\mathrm{AcO})_{4}$ to give aldehyde 19 in excellent overall yield (84\%) from ent-halimic acid 1. In this manner we have opened a new and versatile route to 
intermediate 19, a key compound for the synthesis of many natural products. Once this compound was available in large quantities in a reliable fashion it was decided to synthesize compound 2.

Reaction of 19 with 3-furyl lithium gives 20 and 21, that were separated and charactherised as their acetyl derivatives 22 and 23. The careful hydrolysis in acid medium of 22 and 23 led to ketones $\mathbf{2 4}$ and 25. Alkaline hydrolysis of $\mathbf{2 4}$ gives the hydroxyderivative $\mathbf{2 6}$. Reduction of $\mathbf{2 6}$ with $\mathrm{NaBH}_{4}$ produced 27 and lactone 13. Oxidation of 13 gives the desired ent-halimanolide $2[\alpha]_{\mathbf{D}}^{22}-101.4$ (c 0.2, $\mathrm{CHCl}_{3}$ ). The overall yield for the synthesis of ent-halimanolide 2 from ent-halimic acid $\mathbf{1}$ by route B was 26\%. Compound 2 has been tested against human tumor cell lines: HL-60, $\mathrm{IC}_{50}>10^{-5} \mathrm{M}$, HeLa, $\mathrm{IC}_{50}, 1.6 \pm 0.110^{-5} \mathrm{M}$, A549, $\mathrm{IC}_{50}>10^{-5} \mathrm{M}$, HT-29, $\mathrm{IC}_{50}>10^{-5} \mathrm{M}$. As can be seen, compound 2, is only moderately active against HeLa (human cervix cancer).

\section{Conclusions}

The synthesis of the natural ent-halimanolide $\mathbf{2}$ has been achieved starting from ent-halimic acid $\mathbf{1}$, confirming in this way its structure and establishing its absolute configuration. A new and versatile route to the key intermediate 19 [84\% from 1] for the synthesis of natural ent-halimanolides is described. Other biological tests for $\mathbf{2}$ and several intermediates are in progress and will be reported in due course.

\section{Experimental}

\section{General}

Unless otherwise stated, all chemicals were purchased were of the highest purity commercially available and were used without further purification. IR spectra were recorded on a BOMEM 100 FTIR or an AVATAR 370 FT-IR Thermo Nicolet spectrophotometers. ${ }^{1} \mathrm{H}$ - and ${ }^{13} \mathrm{C}$-NMR spectra were recorded for $\mathrm{CDCl}_{3}$ solutions and referenced to the residual peak of $\mathrm{CHCl}_{3}$ at $\delta 7.26 \mathrm{ppm}$ and $\delta 77.0$ ppm, for ${ }^{1} \mathrm{H}$ and ${ }^{13} \mathrm{C}$, respectively, using Varian $200 \mathrm{VX}$ and Bruker DRX 400 instruments. Chemical shifts are reported in $\delta$ ppm and coupling constants $(J)$ are given in Hz. MS were performed at a VGTS 250 spectrometer at $70 \mathrm{eV}$ ionising voltage. Mass spectra are presented as $\mathrm{m} / \mathrm{z}$ (\% rel. int.). HRMS were recorded on a VG Platform (Fisons) spectrometer using chemical ionization (ammonia as gas) or Fast Atom Bombardment (FAB) technique. For some of the samples, QSTAR XL spectrometer was employed for electrospray ionization (ESI). Optical rotations were determined on a Perkin-Elmer 241 polarimeter in $1 \mathrm{dm}$ cells. Diethyl ether and THF were distilled from sodium, and dichloromethane was distilled from calcium hydride under Ar atmosphere.

Methyl 12-acetoxy-2-ethylenedioxy-13,14,15,16-tetranor-ent-halim-5(10)-en-18-oate (7)

Acetate 6 (1.02 g, $3.28 \mathrm{mmol})$ dissolved in benzene $(33 \mathrm{~mL})$, was refluxed in the presence of p-toluenesulfonic acid (17 mg, $0.10 \mathrm{mmol})$ and ethylene glycol $\left(2.0 \mathrm{~mL}\right.$, excess) at $138{ }^{\circ} \mathrm{C}$ for $16 \mathrm{~h}$, then the solution was diluted with AcOEt, washed with $6 \%$ aqueous $\mathrm{NaHCO}_{3}$ and water and dried over $\mathrm{Na}_{2} \mathrm{SO}_{4}$. The solvent was evaporated to yield 7 (546 mg, 97\%) as a colourless oil; $[\alpha]_{\mathbf{D}}^{22}-19.1$ (c 1.3, 
$\left.\mathrm{CHCl}_{3}\right)$; IR (film) $v\left(\mathrm{~cm}^{-1}\right)$ 1738, 1458, 1373, 1238, 1080, 1032; ${ }^{1} \mathrm{H}-\mathrm{NMR}$ (200 MHz): 4.20-3.80 (6H, m, - $\left.\mathrm{OC}_{2} \mathrm{H}_{4} \mathrm{O}-, \mathrm{H}-12\right), 3.63$ (3H, s, -COOMe), 2.41 (1H, d, $\left.J=13.2 \mathrm{~Hz}, \mathrm{H}_{\mathrm{A}}-3\right), 2.24$ (2H, s, H-1), 2.01 (3H, s, MeCOO-), 1.80-1.50 (4H, m), 1.72 (1H, d, $J=13.2$ Hz, HB -3 ), 1.50-1.20 (3H, m), 1.33 (3H, s, Me-19), 0.88 (3H, s, Me-20), 0.87 (3H, d, $J=6.7 \mathrm{~Hz}, \mathrm{Me}-17) ;{ }^{13} \mathrm{C}-\mathrm{NMR}$ (50 MHz): 177.2 (C-18), 170.9 (MeC-OO-), 132.7 (C-10), 130.9 (C-5), 107.5 (C-2), 64.3/64.1 (-OC $\left.{ }_{2} \mathrm{H}_{4} \mathrm{O}-\right)$, 61.6 (C-12), 51.9 (COOMe), 48.9 (C-4), 42.0 (C-3), 39.8 (C-9), 35.8 (C-1), 35.6 (C-11), 34.1 (C-8), 26.5 (C-7), 24.9 (C6), 23.7 (C-19), 20.9 (C-20), 20.8 (MeCOO-), 15.9 (C-17); HRMS (EI) m/z calcd. for $\mathrm{C}_{21} \mathrm{H}_{32} \mathrm{O}_{6}(\mathrm{M})^{+}$: 380.2199; found 380.2191.

Methyl 12-acetoxy-2-oxo-13,14,15,16-tetranor-ent-halim-5(10)-en-18-oate (8)

To a solution of acetate 7 (48 mg, $0.13 \mathrm{mmol}$ ) in EtOH (2.0 mL), aq. $\mathrm{HCl}$ (2M, $1.7 \mathrm{~mL}$ ) was added. The reaction mixture was stirred for $3 \mathrm{~h}$ at room temperature, then it was diluted with $\mathrm{Et}_{2} \mathrm{O}$, extracted with $\mathrm{Et}_{2} \mathrm{O}$, washed with water and dried over $\mathrm{Na}_{2} \mathrm{SO}_{4}$. Evaporation of the organic layer yielded 8 (41 mg, 92\%) as a colourless oil; $[\alpha]_{\mathrm{D}}^{22}-9.8$ (c 1.0, $\left.\mathrm{CHCl}_{3}\right)$; IR (film) $v\left(\mathrm{~cm}^{-1}\right)$ 2963, 1736, 1680, 1459, 1238, 1033; ${ }^{1} \mathrm{H}-\mathrm{NMR}$ (200 MHz): 4.09-3.95 (1H, m, H-12), 3.83-3.74 (1H, m, H-12), 3.68 (3H, s, COOMe), $2.92\left(1 \mathrm{H}, \mathrm{d}, J=12.2 \mathrm{~Hz}, \mathrm{H}_{\mathrm{A}}-1\right), 2.90\left(1 \mathrm{H}, \mathrm{d}, J=15.0 \mathrm{~Hz}, \mathrm{H}_{\mathrm{A}}-3\right), 2.25(1 \mathrm{H}, \mathrm{d}, J=15.0 \mathrm{~Hz}$,

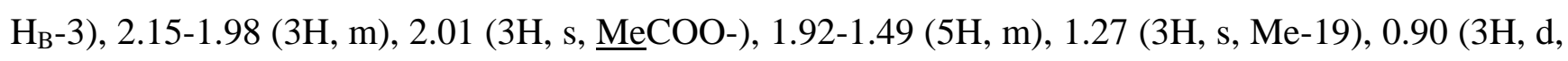
$J=6.6 \mathrm{~Hz}, \mathrm{Me}-17), 0.86$ (3H, s, Me-20); ${ }^{13} \mathrm{C}-\mathrm{NMR}$ (50 MHz): 208.2 (C-2), 174.3 (C-18), 170.7 (MeCOO-), 133.3 (C-10), 133.0 (C-5), 60.7 (C-12), 52.0 (-COOMe), 48.9 (C-1), 48.4 (C-4), 39.4 (C9), 38.8 (C-3), 34.2 (C-11), 33.3 (C-8), 26.0 (C-7), 25.1 (C-6), 21.8 (C-19), 20.5 (C-20), 20.2 (MeCOO-), 15.4 (C-17); HRMS (EI) m/z calcd. for $\mathrm{C}_{19} \mathrm{H}_{28} \mathrm{O}_{5}\left(\mathrm{M}^{+}\right)$: 336.1937; found 336.1928.

12-Acetoxy-13,14,15,16-tetranor-ent-halim-5(10)-en-18,2 $\beta$-olide (10) and methyl 12-acetoxy-2Rhydroxy-13,14,15,16-tetranor-ent-halim-5(10)-en-18-oate (9)

To an ice cooled solution of 8 (0.15 g, $0.43 \mathrm{mmol})$ in EtOH $(4.3 \mathrm{~mL}), \mathrm{NaBH}_{4}(16.2 \mathrm{mg}, 0.43$ $\mathrm{mmol}$ ) was added. After being stirred at room temperature for $3 \mathrm{~h}$, the reaction mixture was recooled to $0^{\circ} \mathrm{C}$ and quenched with a few drops of $2 \mathrm{M}$ aqueous $\mathrm{HCl}$ solution, diluted with EtOAc and water and extracted with EtOAc. The organic layer was washed with water. Evaporation of the dried extract gave a residue which was chromatographed on silica gel (hex/EtOAc 9:1) to afford 10 (73 mg, 49\%) and 9 (70 mg, 47\%). Compound 10: a colourless oil; $[\alpha]_{\mathrm{D}}^{22}-103.1$ (c 0.4, $\mathrm{CHCl}_{3}$ ); IR (film) v ( $\left.\mathrm{cm}^{-1}\right)$ 2959, 1769, 1732, 1456, 1238, 1081,1031; ${ }^{1} \mathrm{H}-\mathrm{NMR}$ (400 MHz): 4.81 (1H, m, H-2), 4.03 (1H, m H-12), 3.84 (1H, m, H-12), 2.61-2.24 (4H, m), 2.15-2.01 (3H, m), 2.02 (3H, s, OCOMe), 1.95- 1.69 (4H, m), 1.30 (3H, s, Me-19), 0.91 (3H, s, Me-20), 0.88 (3H, d, $J=7.0 \mathrm{~Hz}, \mathrm{Me}-17) ;{ }^{13} \mathrm{C}-\mathrm{NMR}$ (100 MHz): 171.10 (MeCOO-), 169.41 (C-18), 133.3 (C-10), 133.0 (C-5), 74.2 (C-2), 60.9 (C-12), 43.4 (C-4), 41.1 (C-1), 39.0 (C-9), 36.1 (C-3), 33.0 (C-8), 31.2 (C-11), 25.9 (C-7), 23.8 (C-6), 21.6 (C-19), 20.8 (MeCOO-), 16.8 (C-20), 20.2 (MeCOO-), 15.5 (C-17); HRMS (EI) m/z calcd. for $\mathrm{C}_{18} \mathrm{H}_{26} \mathrm{O}_{4} \mathrm{Na}$ 329.1723; found 329.1716; Compound 9: a colourless oil; $[\alpha]_{\mathbf{D}}^{22}-88.0$ (c 0.7, $\left.\mathrm{CHCl}_{3}\right)$; IR (film) $v\left(\mathrm{~cm}^{-1}\right)$ 3440, 2938, 1732, 1460, 1368, 1273, 1162, 1045; ${ }^{1} \mathrm{H}-\mathrm{NMR}$ (200 MHz): 4.01 (3H, m, H-2, H-12), 3.64 (3H, s, COOMe), 2.48-2.10 (4H, m), 2.01 (3H, s, MeCOO-), 1.85-1.40 (6H, m), 1.30 (3H, s, Me-19), 0.90 
(3H, s, Me-20), 0.87 (3H, d, $J=7.0 \mathrm{~Hz}, \mathrm{Me}-17) ;{ }^{13} \mathrm{C}-\mathrm{NMR}(50 \mathrm{MHz}): 176.8$ (C-18), 170.7 (MeCOO-), 132.7 (C-10), 130.1 (C-5), 65.0 (C-2), 61.3 (C-12), 51.7 (-COOMe), 48.2 (C-4), 43.9 (C-1), 39.3 (C9), 36.2 (C-3), 33.6 (C-8), 34.2 (C-11), 25.8 (C-7), 24.6 (C-19), 23.4 (C-6), 20.7 (MeCOO-), 20.7 (C20), 20.7 (MeCOO-), 15.4 (C-17); HRMS (EI) m/z calcd. for $\mathrm{C}_{19} \mathrm{H}_{30} \mathrm{O}_{5} \mathrm{Na}$ 361.1985; found 361.1976.

\section{2-Hydroxy-13,14,15,16-tetranor-ent-halim-5(10)-en-18,2 $\beta$-olide (11)}

To 10 (73.0 mg, $0.24 \mathrm{mmol}$ ) a $3 \%$ solution of $\mathrm{K}_{2} \mathrm{CO}_{3}$ in methanol (5 ml) was added. After $2 \mathrm{~h}$ the solvent was evaporated and diluted with $\mathrm{Et}_{2} \mathrm{O}$. The organic layer was successively washed with a $2 \mathrm{~N}$ aqueous solution of $\mathrm{HCl}$ and water, dried over $\mathrm{Na}_{2} \mathrm{SO}_{4}$ and evaporated to yield the expected compound 11 (61 mg, 97\%) as a colourless oil; [ $\alpha]_{\mathbf{D}}^{22}-105.5$ (c 0.4, $\mathrm{CHCl}_{3}$ ); IR (film) $v\left(\mathrm{~cm}^{-1}\right.$ ) 3419, 2938, 1770, 1457, 1381, 1189, 1162, 948; ${ }^{1} \mathrm{H}-\mathrm{NMR}$ (200 MHz): 4.82 (1H, m, H-2), 3.67-3.48 (1H, m, $\left.\mathrm{H}_{\mathrm{A}}-12\right)$, 3.42-3.28 (1H, m, HB-12), 2.45-2.05 (5H, m), 1.96-1.43 (5H, m), 1.28 (3H, s, Me-19), 0.88 (3H, s, Me20), 0.86 (3H, d, $J=6.1 \mathrm{~Hz}, \mathrm{Me}-17) ;{ }^{13} \mathrm{C}-\mathrm{NMR}$ (50 MHz): 179.2 (C-18), 134.0 (C-10), 133.3 (C-5), 74.6 (C-2), 59.2 (C-12), 43.7 (C-4), 41.3 (C-1), 40.3 (C-3), 39.3 (C-9), 33.7 (C-8), 31.4 (C-11), 26.4 (C-6), 24.5 (C-7), 21.8 (C-20), 17.1 (C-19), 16.0 (C-17); HRMS (EI) m/z calcd. for $\mathrm{C}_{16} \mathrm{H}_{24} \mathrm{O}_{3} \mathrm{Na}$ 287.1618; found 287.1612.

\section{2-Oxo-13,14,15,16-tetranor-ent-halim-5(10)-en-18,2 $\beta$-olide (12)}

To a mixture of 11 (32 mg, $0.12 \mathrm{mmol}$ ) $N$-methylmorpholine- $N$-oxide (NMO) (49 mg, $0.36 \mathrm{mmol}$ ) and molecular sieves (60 mg, $500 \mathrm{mg} / \mathrm{mmol}$ ) in anhydrous $\mathrm{CH}_{2} \mathrm{Cl}_{2}(1.2 \mathrm{~mL}$ ) under an Ar atmosphere and at room temperature, TPAP ( $2 \mathrm{mg}, 5 \times 10^{-3} \mathrm{mmol}$ ) was added. The reaction mixture was stirred for 30 min. and then filtered on silica gel and Celite (DCM and EtOAc). Evaporation of the solvent yielded 12 (30 mg, 96\%) as a colourless oil, IR (film) $v\left(\mathrm{~cm}^{-1}\right)$ 2937, 1770, 1719, 1460, 1189,1078,948; ${ }^{1} \mathrm{H}-\mathrm{NMR}$ (200 MHz): 9.56 (1H, m, H-12), 4.80 (1H, m, H-2), 2.48-2.41 (3H, m), 2.192.14 (2H, m), 1.96 (1H, d, $\left.J=13.0 \mathrm{~Hz}, \mathrm{H}-11_{\mathrm{B}}\right), 1.77-1.20$ (5H, m), 1.30 (3H, s, Me-19), 1.02 (3H, s, Me-20), 0.90 (3H, d, $J=6.6 \mathrm{~Hz}$, Me-17); HRMS (EI) m/z calcd. for $\mathrm{C}_{16} \mathrm{H}_{22} \mathrm{O}_{3} \mathrm{Na} 285.1461$; found 281.1476 .

15,16-Epoxy-12S-hidroxy-ent-halima-5(10),13(16),14-trien-18,2 $\beta$-olide (13) and 15,16-epoxy-12Rhydroxy-ent-halima-5(10),13(16),14-trien-18,2 $\beta$-olide (14)

A solution of 3-bromofuran in THF $1 \mathrm{M}(0.14 \mathrm{~mL}, 0.14 \mathrm{mmol})$, was treated dropwise with $n$-BuLi (1.6 $\mathrm{M}$ in hexane, $0.09 \mathrm{~mL}, 0.14 \mathrm{mmol}$ ) at $-78^{\circ} \mathrm{C}$ under $\mathrm{Ar}$ atmosphere. After the reaction mixture was stirred for $30 \mathrm{~min}$. at this temperature, a solution of 12 (30 mg, $0.11 \mathrm{mmol})$ in dry THF $(1.1 \mathrm{~mL})$ was added and stirred for an additional 30 minutes at the same temperature. The reaction mixture was treated with $10 \%$ aqueous $\mathrm{NH}_{4} \mathrm{Cl}$ solution, warmed to room temperature and extracted with EtOAc. The organic layer was washed with $6 \% \mathrm{NaHCO}_{3}$, brine and dried over $\mathrm{Na}_{2} \mathrm{SO}_{4}$. The solvent was evaporated to afford a residue which was purified by chromatography (Hex/AcOEt 9/1) to yield 13 (20 mg, 54\%) and 14 (13 mg, 36\%). Compound 13: colourless oil; $[\alpha]_{\mathbf{D}}^{22}-31.1$ (c $\left.0.4, \mathrm{CHCl}_{3}\right)$. IR (film) v $\left(\mathrm{cm}^{-1}\right)$ 3400, 2927, 1769, 1460, 1070, 1024. ${ }^{1} \mathrm{H}-\mathrm{NMR}$ (200 MHz): 7.39 (1H, s, H-15), 7.36 (1H, s, H- 
16), 6.40 (1H, d, $J=2.20 \mathrm{~Hz}, \mathrm{H}-14), 4.76$ (1H, dd, $J=5.6,2.7 \mathrm{~Hz}, \mathrm{H}-2), 4.43$ (1H, d, $J=7.9 \mathrm{~Hz}, \mathrm{H}-$ 12), 2.38-1.93 (7H, m), 1.91-1.42 (4H, m), 1.30 (3H, s, Me-19), 0.92 (3H, d, $J=6.6 \mathrm{~Hz}, \mathrm{Me}-17$ ), 0.91(3H, s, Me-20). ${ }^{13} \mathrm{C}-\mathrm{NMR}$ (50 MHz): 178.6 (C-18),143.5 (C-16), 138.5 (C-15), 134.0 (C-10), 133.6 (C-5), 130.9 (C-13), 108.0 (C-14), 74.5 (C-2), 64.0 (C-12), 45.3 (C-11), 43.3 (C-4), 41.0 (C-3), 40.4 (C-9), 33.0 (C-8), 31.1 (C-1), 26.0 (C-7), 24.1 (C-6), 21.1 (C-20), 16.9 (C-19), 15.8 (C-17); HRMS (EI) $\mathrm{m} / \mathrm{z}$ calcd. for $\mathrm{C}_{20} \mathrm{H}_{26} \mathrm{O}_{4} \mathrm{Na}$ : 353.1723; found 353.1718. Compound 14: colourless oil; $[\alpha]_{\mathbf{D}}^{22}-63.1$ (с 0.3, $\mathrm{CHCl}_{3}$ ); IR (film) $v\left(\mathrm{~cm}^{-1}\right)$ 3407, 2927, 1769, 1460, 1075, 1024; ${ }^{1} \mathrm{H}-\mathrm{NMR}(200$ MHz): 7.40 (1H, d, $J=1.6$ Hz, H-15), 7.36 (1H, s, H-16), 6.41 (1H, dd, $J=2.13,0.61 \mathrm{~Hz}, \mathrm{H}-14), 4.76$ (1H, ddd, $J=5.6,2.9,2.7 \mathrm{~Hz}, \mathrm{H}-2), 4.44$ (1H, dd, $J=7.7,1.7$ Hz, H-12), 2.34-1.93 (7H, m), 1.90-1.40 (4H, m), 1.32 (3H, s, Me-19), 0.94 (3H, d, $J=6.8 \mathrm{~Hz}, \mathrm{Me}-17), 0.93$ (3H, s, Me-20); ${ }^{13} \mathrm{C}-\mathrm{NMR}(50$ MHz): 178.7 (C-18),143.5 (C-16), 138.7 (C-15), 134.0 (C-10), 133.7 (C-5), 130.7 (C-13), 108.2 (C14), 74.3 (C-2), 64.2 (C-12), 45.6 (C-11), 43.5 (C-4), 41.2 (C-3), 40.5 (C-9), 33.1 (C-8), 31.4 (C-1), 26.2 (C-7), 24.3 (C-6), 21.4 (C-20), 16.9 (C-19), 15.8 (C-17); HRMS (EI) m/z calcd. for $\mathrm{C}_{20} \mathrm{H}_{26} \mathrm{O}_{4} \mathrm{Na}$ : 353.1723; found 353.1712 .

\section{5,16-Epoxy-12-oxo-ent-halima-5(10),13(16),14-trien-18,2 $\beta$-olide (2)}

To a mixture of 13/14 (3 mg, $0.01 \mathrm{mmol}$ ) $N$-methylmorpholine- $N$-oxide (NMO, $4 \mathrm{mg}, 0.03 \mathrm{mmol}$ ) and molecular sieves $(10 \mathrm{mg})$ in anhydrous $\mathrm{CH}_{2} \mathrm{Cl}_{2}(0.3 \mathrm{~mL})$ under an $\mathrm{Ar}$ atmosphere and at room temperature, TPAP (1.0 mg, $3 \times 10^{-3} \mathrm{mmol}$ ) was added. The reaction mixture was stirred for $30 \mathrm{~min}$. and then filtered on silica gel and Celite (DCM and EtOAc). Evaporation of the solvent yielded 2 (3.0 mg, 92\%) as a colourless oil; $[\alpha]_{\mathrm{D}}^{22}-101.4$ (c 0.2, $\left.\mathrm{CHCl}_{3}\right)$; IR (film) $\vee\left(\mathrm{cm}^{-1}\right)$ 2928, 1733, 1240, 1077, 1023; ${ }^{1} \mathrm{H}-\mathrm{NMR}$ (200 MHz): 7.94 (1H, dd, $\left.J=1.6,0.8 \mathrm{~Hz}, \mathrm{H}-16\right), 7.41$ (1H, dd, $J=12.0,1.6 \mathrm{~Hz}, \mathrm{H}-$ 15), $6.73(1 \mathrm{H}, \mathrm{dd}, J=1.6,0.8 \mathrm{~Hz}, \mathrm{H}-14), 4.76$ (1H, ddd, $J=5.6,2.8,2.8 \mathrm{~Hz}, \mathrm{H}-2), 2.85(1 \mathrm{H}, \mathrm{d}, J=$ $\left.15.6 \mathrm{~Hz}, \mathrm{H}_{\mathrm{A}}-11\right)$, 2.74 (1H, d, $\left.J=15.6 \mathrm{~Hz}, \mathrm{H}_{\mathrm{B}}-11\right)$, 2.39-2.35 (2H, m, H-1), 2.19-2.10 (2H, m, H-6), $2.13\left(1 \mathrm{H}, \mathrm{dd}, J=10.8,6.8 \mathrm{~Hz}, \mathrm{H}_{\mathrm{A}}-3\right)$, 2.08-2.04 (1H, m, H-8), 1.94 (1H, d, $\left.J=10.8 \mathrm{~Hz}, \mathrm{H}_{\mathrm{B}}-3\right), 1.81-$ 1.42 (2H, m, H-7), 1.32 (3H, s, Me-19), 1.09 (3H, s, Me-20), 0.86 (3H, d, $J=7.0 \mathrm{~Hz}, \mathrm{Me}-17) ;{ }^{13} \mathrm{C}-$ NMR (50 MHz): 193.5 (C-12), 178.2 (C-18), 146.9 (C-16), 144.1 (C-15), 132.4 (C-10), 132.1 (C-5), 129.3 (C-13), 108.7 (C-14), 73.9 (C-2), 47.7 (C-11), 43.5 (C-4), 41.1 (C-3), 40.3 (C-9), 33.2 (C-8), 31.6 (C-1), 25.2 (C-7), 22.1 (C-6), 21.8 (C-20), 16.4 (C-19), 15.1 (C-17); HRMS (EI) m/z calcd. for $\mathrm{C}_{20} \mathrm{H}_{24} \mathrm{O}_{4} \mathrm{Na}$ : 351.1567; found 351.1567.

\section{Methyl 2-oxo-15-nor-ent-halima-1(10),13(14)-dien-18-oate (16)}

To a suspension of $\mathrm{MeOCH}_{2} \mathrm{PPh}_{3} \mathrm{Cl}(1.24 \mathrm{~g}, 3.15 \mathrm{mmol})$ in THF $(10 \mathrm{~mL})$ at $-20^{\circ} \mathrm{C}$ under an $\mathrm{Ar}$ atmosphere, 1.0 M NaHMDS in THF (3.15 mL, $3.15 \mathrm{mmol}$ ) was added dropwise and the solution was stirred for $30 \mathrm{~min}$. A solution of the aldehyde 15 (1.0 g, $3.12 \mathrm{mmol})$ in THF (15 mL) was added dropwise at $-78^{\circ} \mathrm{C}$. The mixture was stirred for $1 \mathrm{~h}$ at room temperature. Then, it was quenched with aqueous $\mathrm{NH}_{4} \mathrm{Cl}$ and extracted with AcOEt. The organic layer was washed with brine and dried over $\mathrm{Na}_{2} \mathrm{SO}_{4}$. The solvent was evaporated to afford a residue which was purified by chromatography (Hex/ AcOEt 97/3) to yield 16 (933 mg, 94\%) as a colourless oil; $[\alpha]_{\mathbf{D}}^{22}+179.9$ (c 1.4, $\mathrm{CHCl}_{3}$ ); IR (film) v 
$\left(\mathrm{cm}^{-1}\right)$ 2964, 1732, 1676, 1455, 1267, 1160, 1113, 883; ${ }^{1} \mathrm{H}-\mathrm{NMR}$ (200 MHz): 5.81 (1H, s, H-1), 4.68 (2H, s, H-14), 3.60 (3H, s, -COOMe), 3.00 (1H, dd, $J=12.1,4.7 \mathrm{~Hz}, \mathrm{H}-5), 2.66$ (1H, d, $J=16.2 \mathrm{~Hz}$, H-3 ${ }_{\mathrm{A}}$ ), 2.24 (1H, d, $\left.J=16.2 \mathrm{~Hz}, \mathrm{H}-3_{\mathrm{B}}\right), 2.18-1.97$ (3H, m), 1.83-1.36 (6H, m), 1.70 (3H, s, Me-16), 1.20 (3H, s, Me-19), 0.98 (3H, s, Me-20), 0.78 (3H, d, $J=7.4 \mathrm{~Hz}, \mathrm{Me}-17) ;{ }^{13} \mathrm{C}-\mathrm{NMR}$ (50 MHz): 196.3 (C-2), 175.9 (C-18) 168.7 (C-10), 145.2 (C-13), 124.2 (C-1), 108.9 (C-14), 51.6 (-COOMe), 45.5 (C4), 44.4 (C-9), 42.2 (C-3), 40.2 (C-5), 39.9 (C-8), 36.4 (C-12), 31.1 (C-11), 27.5 (C-7), 22.8 (C-6), 21.9 (C-19), 21.2 (C-16), 20.0 (C-20), 14.8 (C-17); HRMS (EI) m/z calcd. for $\mathrm{C}_{20} \mathrm{H}_{30} \mathrm{O}_{3} \mathrm{Na}$ : 341.2087; found 341.2073 .

Methyl 2-oxo-15-nor-ent-halima-1(10),12(13)-dien-18-oate (17)

To a solution of 16 (1.06 g, $3.35 \mathrm{mmol})$ in benzene (33 $\mathrm{mL}), p$-TsOH $(0.16 \mathrm{~g}, 0.91 \mathrm{mmol})$ was added. The reaction mixture was stirred at $60^{\circ} \mathrm{C}$ for $2 \mathrm{~h}$, then it was cooled and diluted with $\mathrm{Et}_{2} \mathrm{O}$. The organic layer was washed with $6 \%$ aqueous $\mathrm{NaHCO}_{3}$ and brine and dried over $\mathrm{Na}_{2} \mathrm{SO}_{4}$. The solvent was evaporated to yield 17 (1.01 g, 96\%) as a colourless oil; $[\alpha]_{\mathbf{D}}^{22}+45.4$ (c 1.0, $\mathrm{CHCl}_{3}$ ); IR (film) v $\left(\mathrm{cm}^{-1}\right)$ 2929, 1732, 1676, 1457, 1269, 1116; ${ }^{1} \mathrm{H}-\mathrm{NMR}(200 \mathrm{MHz}): 5.74$ (1H, s, H-1), 4.71 (1H, m, H12), 3.57 (3H, s, -COOMe), 3.00 (1H, dd, $J=12.4,4.5 \mathrm{~Hz}, \mathrm{H}-5), 2.63$ (1H, d, $J=16.0 \mathrm{~Hz}, \mathrm{H}-3_{\mathrm{A}}$ ), 2.19 (1H, d, $J=15.6$ H-3 B , 2.10-1.90 (3H, m), 1.85- 1.22 (5H, m), 1.53 (3H, s, Me-16), 1.47 (3H, s, Me14), 1.16 (3H, s, Me-19), 0.86 (3H, s, Me-20), 0.72 (3H, d, $J=7.0 \mathrm{~Hz}, \mathrm{Me}-17) ;{ }^{13} \mathrm{C}-\mathrm{NMR}(50 \mathrm{MHz})$ : 197.4 (C-2), 176.8 (C-18) 169.8 (C-10), 133.9 (C-13), 124.9 (C-1), 120.0 (C-12), 52.5 (-COOMe), 46.5 (C-4), 46.1 (C-9), 43.6 (C-3), 41.2 (C-5), 40.3 (C-8), 37.7 (C-11), 28.6 (C-7), 26.2 (C-20), 23.7 (C-6), 21.7 (C-19), 21.7 (C-16), 18.2 (C-14), 15.8 (C-17); HRMS (EI) m/z calcd. for $\mathrm{C}_{20} \mathrm{H}_{30} \mathrm{O}_{3} \mathrm{Na}$ : 341.2087; found 341.2097.

Methyl 2-ethylenedioxy-15-nor-ent-halima-5(10),12(13)-dien-18-oate (18)

Compound 17 ( $1 \mathrm{~g}, 3.15 \mathrm{mmol}$ ) dissolved in benzene (32 mL), was refluxed in the presence of p-toluenesulfonic acid (19 mg, $0.11 \mathrm{mmol})$ and ethylene glycol $\left(1.9 \mathrm{ml}\right.$, excess) at $138^{\circ} \mathrm{C}$ for $16 \mathrm{~h}$. The solution was then diluted with AcOEt and washed with $6 \%$ aqueous $\mathrm{NaHCO}_{3}$ and water and dried over $\mathrm{Na}_{2} \mathrm{SO}_{4}$. The solvent was evaporated to yield $18(1.11 \mathrm{~g}, 97 \%)$ as a colourless oil; $[\alpha]_{\mathbf{D}}^{22}-20.3$ (c 1.4, $\mathrm{CHCl}_{3}$ ); IR (film) $v\left(\mathrm{~cm}^{-1}\right)$ 2964, 1733, 1237, 1118, 1078; ${ }^{1} \mathrm{H}-\mathrm{NMR}$ (200 MHz): 4.98 (1H, m, H-12), $3.96\left(4 \mathrm{H}, \mathrm{m},-\mathrm{OC}_{2} \mathrm{H}_{4} \mathrm{O}-\right)$, 3.64 (3H, s, -COOMe), 2.31 (2H, s, H-3), 2.20-1.95 (4H, m), 1.92-1.30(5H, m), 1.66 (3H, s, Me-16), 1.57 (3H, s, Me-14), 1.33 (3H, s, Me-19), 0.84 (3H, s, Me-20), 0.80 (3H, d, $J$ = $7.0 \mathrm{~Hz}, \mathrm{Me}-17)$; ${ }^{13} \mathrm{C}-\mathrm{NMR}$ (50 MHz): 176.1 (C-18), 132.5 (C-13), 131.0 (C-5), 129.2 (C-10), 120.6 (C-12), 106.7 (C-2), 63.2 (-OC $\left.\mathrm{H}_{4} \mathrm{O}-\right)$, 63.0 (-OC $\left.\mathrm{H}_{4} \mathrm{O}-\right)$, 50.7 (-COOMe), 48.0 (C-4), 40.8 (C-3), 39.8 (C-9), 34.8 (C-1), 34.7 (C-11), 32.8 (C-8), 25.6 (C-7), 25.1 (C-20), 23.9 (C-6), 22.6 (C-16), 19.4 (C19), 17.0 (C-14), 15.2 (C-17); HRMS (EI) m/z calcd. for $\mathrm{C}_{22} \mathrm{H}_{34} \mathrm{O}_{4} \mathrm{Na}$ : 385.2349; found 385.2362.

Methyl 2-ethylenedioxy-12-oxo-13,14,15,16-tetranor-ent-halim-5(10)-en-18-oate (19).

To a solution of $18(1.05 \mathrm{~g}, 2.90 \mathrm{mmol})$ in $t-\mathrm{BuOH} / \mathrm{THF} / \mathrm{H}_{2} \mathrm{O}(7: 2: 1,30.5 \mathrm{ml})$ was added $N$-methylmorpholine $N$-oxide (NMO, $1.18 \mathrm{~g}, 8.70 \mathrm{mmol})$ and a solution of $\mathrm{OsO}_{4} 2.5 \%(0.3 \mathrm{ml}, 0.01 \mathrm{mmol})$ in 
$t$ - $\mathrm{BuOH}$. The reaction mixture was stirred at room temperature for $20 \mathrm{~h}$ and a saturated aqueous solution of $\mathrm{Na}_{2} \mathrm{SO}_{3}(30 \mathrm{~mL})$ was added. The mixture was extracted with AcOEt, and the organic layer was washed with $10 \%$ aqueous $\mathrm{Na}_{2} \mathrm{~S}_{2} \mathrm{O}_{3}, 2 \mathrm{~N}$ aqueous $\mathrm{HCl}$, water and brine and dried over $\mathrm{Na}_{2} \mathrm{SO}_{4}$. The solvent was evaporated to yield the expected mixture of hydroxy derivatives. To a solution of the hydroxy derivatives $(1.15 \mathrm{~g}, 3.06 \mathrm{mmol})$ in benzene $(16 \mathrm{ml})$ was added LTA (3.0 g, $6.68 \mathrm{mmol})$. The reaction mixture was stirred at room temperature for $30 \mathrm{~min}$ and then filtered off through Celite. The solution was diluted with EtOAc and washed with $6 \%$ aqueous $\mathrm{NaHCO}_{3}$, water and brine and then dried and evaporated to yield 19 (980 mg, 96\%) as a colourless oil; $[\alpha]_{\mathbf{D}}^{22}+2.1$ (c 0.9, $\mathrm{CHCl}_{3}$ ); IR (film) $v\left(\mathrm{~cm}^{-1}\right)$ 1734, 1717, 1456, 1375, 1238, 1152, 1078, 1032; ${ }^{1} \mathrm{H}-\mathrm{NMR}$ (200 MHz): 9.63 (1H, s, H-12), 4.00-3.80 (4H, m, - $\mathrm{OC}_{2} \mathrm{H}_{4} \mathrm{O}-$ ), 3.60 (3H, s, -COOMe), 2.60-2.20 (5H, m, H-11, H-1, $\mathrm{H}_{\mathrm{A}}-3$ ), 2.10-1.90 (1H, m), 1.62 (1H, d, $\left.J=13.2 \mathrm{~Hz}, \mathrm{H}_{\mathrm{B}}-3\right), 1.80-1.60$ (2H, m), 1.40-1.20 (2H, m), 1.31 (3H, s, Me-19), 0.96 (3H, s, Me-20), 0.88 (3H, d, $J=6.7 \mathrm{~Hz}, \mathrm{Me}-17)$; ${ }^{13} \mathrm{C}-\mathrm{NMR}$ (50 MHz): 204.3 (C-12), 177.0 (C18), 131.7 (C-10), 131.3 (C-5), 107.2 (C-2), 64.3/64.1 (-OC $\left.\mathrm{H}_{4} \mathrm{O}-\right)$ ), 51.9 (-COOMe), 51.2 (C-11), 48.8 (C-4), 42.2 (C-3), 40.1 (C-9), 36.4 (C-1), 36.4 (C-8), 26.2 (C-7), 24.5 (C-6), 23.9 (C-19), 21.1 (C-20), 15.7 (C-17); HRMS (EI) m/z calcd. for $\mathrm{C}_{19} \mathrm{H}_{28} \mathrm{O}_{5}(\mathrm{M})^{+}:$336.1937; found 336.1916.

Methyl 15,16-epoxy-2-ethylenedioxy-12S-hydroxy-ent-halima-5(10),13(16),14-trien-18-oate (20) and methyl 15,16-epoxy-2- ethylenedioxy -12R-hydroxy-ent-halima-5(10),13(16),14-trien-18-oate (21)

A solution of 3-bromofuran in THF $0.89 \mathrm{M}(1.45 \mathrm{~mL}, 1.28 \mathrm{mmol})$, was treated dropwise with $n$-BuLi (1.6 $\mathrm{M}$ in hexane, $0.85 \mathrm{ml}, 1.35 \mathrm{mmol})$ at $-78^{\circ} \mathrm{C}$ under an Ar atmosphere. After the reaction mixture was stirred for 30 minutes at this temperature, a solution of 19 (430 mg, $1.28 \mathrm{mmol}$ ) in dry THF (1.1 mL) was added and stirred for an additional $30 \mathrm{~min}$. at the same temperature. The reaction mixture was then treated with $10 \%$ aqueous $\mathrm{NH}_{4} \mathrm{Cl}$ solution, warmed to room temperature and extracted with EtOAc. The organic layer was washed with $6 \% \mathrm{NaHCO}_{3}$, brine and dried over $\mathrm{Na}_{2} \mathrm{SO}_{4}$. The solvent was evaporated to afford a residue which was purified by chromatography (hex/AcOEt 95/5) to yield 20 (279 mg, 54\%) and 21 (202 mg, 39\%). Compound 20: a colourless oil; $[\alpha]_{\mathbf{D}}^{22}+4.6$ (c $\left.0.8, \mathrm{CHCl}_{3}\right)$; IR (film) $v\left(\mathrm{~cm}^{-1}\right)$ 3500, 1724, 1458, 1375, 1262, 1157, 1078, 1030, 665; ${ }^{1} \mathrm{H}-\mathrm{NMR}(200$ MHz): 7.34 (2H, s, H-15, H-16), 6.39 (1H, s, H-14), 4.85 (1H, dd, $J=9.0,2.2$ Hz, H-12), 4.00-3.80 (4H, m, $-\mathrm{OC}_{2} \mathrm{H}_{4} \mathrm{O}-$ ), 3.67 (3H, s, -COOMe), 2.38 (1H, d, $\left.J=12.5 \mathrm{~Hz}, \mathrm{H}_{\mathrm{A}}-3\right), 2.35(1 \mathrm{H}, \mathrm{d}, J=10.5 \mathrm{~Hz}$, $\left.\mathrm{H}_{\mathrm{A}}-1\right), 2.15\left(1 \mathrm{H}, \mathrm{d}, J=10.5 \mathrm{~Hz}, \mathrm{H}_{\mathrm{B}}-1\right), 2.00$ (1H, dd, $\left.J=15.0,9.4 \mathrm{~Hz}, \mathrm{H}_{\mathrm{A}}-11\right), 1.72(1 \mathrm{H}, \mathrm{dd}, J=15.0$, $\left.2.2 \mathrm{~Hz}, \mathrm{H}_{\mathrm{B}}-11\right)$, 1.71-1.68 (2H, m, H-6), 1.70 (1H, d, $J=12.5$ Hz, H -3), 1.63-1.56 (1H, m, H-8), 1.371.27 (2H, m, H-7), 1.35 (3H, s, Me-19), 0.93 (3H, s, Me-20), 0.92 (3H, d, J=6.8 Hz, Me-17); ${ }^{13} \mathrm{C}-$ NMR (50 MHz): 177.6 (C-18), 143.0 (C-16), 138.2 (C-15), 133.2 (C-10), 131.7 (C-5), 130.8 (C-13), 108.6 (C-14), 107.3 (C-2), 64.4 (C-12), 64.4/64.2 (-OC $\left.\mathrm{H}_{4} \mathrm{O}-\right)$, 52.0 (-COOMe), 48.6 (C-4), 46.5 (C11), 42.7 (C-3), 40.6 (C-9), 36.7 (C-1), 35.3 (C-8), 26.8 (C-7), 25.2 (C-6), 24.5 (C-19), 21.3 (C-20), 16.1 (C-17); HRMS (EI) m/z calcd for $\mathrm{C}_{23} \mathrm{H}_{32} \mathrm{O}_{6}(\mathrm{M})^{+}:$404.2199; found 404.2191. Compound 21: a colourless oil; $[\alpha]_{\mathbf{D}}^{22}-17.2$ (c 0.5, $\mathrm{CHCl}_{3}$ ); IR (film) $v\left(\mathrm{~cm}^{-1}\right)$ 3500, 1730, 1464, 1377, 1159, 1089, 1030, 665; ${ }^{1} \mathrm{H}-\mathrm{NMR}$ (200 MHz): 7.38 (2H, s, H-15, H-16), 6.38 (1H, s, H-14), 4.91 (1H, dd, $J=9.4,2.3 \mathrm{~Hz}$, $\mathrm{H}-12$ ), 4.00-3.80 (4H, m, - $\mathrm{OC}_{2} \mathrm{H}_{4} \mathrm{O}-$ ), 3.65 (3H, s, -COOMe), 2.60-2.00 (5H, m), 1.90-1.60 (3H, m), 1.40-1.20 (3H, m), 1.36 (3H, s, Me-19), 0.91 (3H, s, Me-20), 0.89 (3H, d, $J=6.9 \mathrm{~Hz}, \mathrm{Me}-17$ ); ${ }^{13} \mathrm{C}-$ 
NMR (50 MHz): 177.6 (C-18), 143.0 (C-16), 138.4 (C-15), 133.4 (C-10), 132.0 (C-5), 130.0 (C-13), 108.7 (C-14), 107.4 (C-2), 64.6/64.3 (-OC ${ }_{2} \mathrm{H}_{4} \mathrm{O}-$ ), 64.2 (C-12), 52.1 (-COOMe), 48.7 (C-4), 47.7 (C11), 42.9 (C-3), 40.6 (C-9), 36.1 (C-1), 35.8 (C-8), 26.9 (C-7), 25.1 (C-6), 24.5 (C-19), 21.8 (C-20), 16.4 (C-17), HRMS (EI) m/z calcd. for $\mathrm{C}_{23} \mathrm{H}_{32} \mathrm{O}_{6}(\mathrm{M})^{+}: 404.2199$; found 404.2193.

Methyl 12S-acetoxy-15,16-epoxy-2-ethylenedioxy-ent-halima-5(10),13(16),14-trien-18-oate (22)

To a solution of 20 (118 mg, $0.29 \mathrm{mmol})$ in dry pyridine $(1.0 \mathrm{~mL}), \mathrm{Ac}_{2} \mathrm{O}(1.0 \mathrm{~mL})$ was added and the mixture was stirred at room temperature overnight, then the reaction mixture was poured into icewater and extracted with EtOAc. The organic layer was washed successively with $2 \mathrm{M}$ aqueous $\mathrm{HCl}$, $6 \%$ aqueous $\mathrm{NaHCO}_{3}$ and brine. It was dried over $\mathrm{Na}_{2} \mathrm{SO}_{4}$ and the solvent was evaporated to afford 22 (128 mg, 99\%) as a colourless oil; $[\alpha]_{\mathrm{D}}^{22}-13.3$ (c 0.7, $\mathrm{CHCl}_{3}$ ); IR (film) $v\left(\mathrm{~cm}^{-1}\right)$ 1733, 1458, 1374, 1240, 1102, 1077, 1023, 874; ${ }^{1} \mathrm{H}-\mathrm{NMR}$ (200 MHz): 7.36 (1H, s, H-16), 7.31 (1H, s, H-15), 6.36 (1H, s, $\mathrm{H}-14), 5.71$ (1H, dd, $J=7.8,2.2 \mathrm{~Hz}, \mathrm{H}-12), 4.00-3.80$ (4H, m, - $\mathrm{OC}_{2} \mathrm{H}_{4} \mathrm{O}-$ ), 3.69 (3H, s, -COOMe), 2.25 (1H, d, $J=13.2 \mathrm{~Hz}, \mathrm{H}_{\mathrm{A}}-3$ ), 2.19-1.99 (4H, m), 1.96 (3H, s, -OCOMe), 1.95-1.39 (5H, m), 1.66 (1H, d, $J=13.2 \mathrm{~Hz}, \mathrm{H}_{\mathrm{B}}-3$ ), 1.32 (3H, s, Me-19), 0.89 (3H, d, $J=6.9 \mathrm{~Hz}, \mathrm{Me}-17$ ), 0.87 (3H, s, Me-20);

${ }^{13} \mathrm{C}-\mathrm{NMR}$ (50 MHz): 177.6 (C-18), 170.2 (-OCOOMe), 143.3 (C-16), 140.2 (C-15), 132.9 (C-10), 131.5 (C-5), 126.7 (C-13), 109.0 (C-14), 107.7 (C-2), 66.4 (C-12), 64.5/64.3 (-OC $\left.\mathrm{H}_{4} \mathrm{O}-\right), 52.2$ (-COOMe), 49.0 (C-4), 42.1 (C-3), 41.9 (C-11), 41.1 (C-9), 36.2 (C-1), 33.8 (C-8), 26.4 (C-7), 25.1 (C-6), 24.0 (C19), 21.6 (-OCOMe), 20.8 (C-20), 16.0 (C-17); HRMS (EI) m/z calcd. for $\mathrm{C}_{25} \mathrm{H}_{34} \mathrm{O}_{7} \mathrm{Na}$ : 469.2197; found 469.2197.

Methyl 12R-acetoxy-15,16-epoxy-2-ethylenedioxy-ent-halima-5(10),13(16),14-trien-18-oate (23)

To a solution of 21 (22 mg, $0.05 \mathrm{mmol})$ in dry pyridine $(0.5 \mathrm{~mL}), \mathrm{Ac}_{2} \mathrm{O}(0.5 \mathrm{~mL})$ was added and the mixture was stirred at room temperature overnight. The reaction mixture was then poured into icewater and extracted with EtOAc. The organic layer was washed successively with $2 \mathrm{M}$ aqueous $\mathrm{HCl}$, $6 \%$ aqueous $\mathrm{NaHCO}_{3}$ and brine. It was dried over $\mathrm{Na}_{2} \mathrm{SO}_{4}$ and the solvent was evaporated to afford 23 (128 mg, 99\%) as a colourless oil; $[\alpha]_{\mathbf{D}}^{22}-51.8$ (c 0.6, $\mathrm{CHCl}_{3}$ ); IR (film) v ( $\left.\mathrm{cm}^{-1}\right)$ 1733, 1458, 1374, 1240, 1102, 1077, 1023, 874; ${ }^{1} \mathrm{H}-\mathrm{NMR}$ (200 MHz): 7.39 (1H, s, H-16), 7.34 (1H, s, H-15), 6.39 (1H, s, $\mathrm{H}-14), 5.89$ (1H, dd, $J=8.8,4.4 \mathrm{~Hz}, \mathrm{H}-12), 3.96-3.80\left(4 \mathrm{H}, \mathrm{m},-\mathrm{OC}_{2} \mathrm{H}_{4} \mathrm{O}-\right), 3.66$ (3H, s, -COOMe), 2.60-2.00 (5H, m), 2.19-1.99 (4H, m), 2.02 (3H, s, -OCOMe), 1.93-1.62 (3H, m), 1.40-1.20 (3H, m), 1.36 (3H, s, Me-19), 0.87 (3H, s, Me-20), 0.82 (3H, d, $J=6.6 \mathrm{~Hz}, \mathrm{Me}-17) ;{ }^{13} \mathrm{C}-\mathrm{NMR}$ (50 MHz): 177.5 (C-18), 170.5 (-OCOMe), 143.3 (C-16), 140.4 (C-15), 133.3 (C-10), 129.7 (C-5), 126.6 (C-13), 109.1 (C-14), 107.9 (C-2), 65.4 (C-12), 64.6/64.2 (-OC $\left.\mathrm{H}_{4} \mathrm{O}-\right)$, 52.2 (-COOMe), 49.5 (C-4), 41.4 (C-3), 41.2 (C-11), 40.9 (C-9), 36.4 (C-1), 33.4 (C-8), 25.6 (C-7), 25.1 (C-6), 23.6 (C-19), 22.0 (-OCOMe), 21.4 (C-20), 16.1 (C-17); HRMS (EI) m/z calcd. for $\mathrm{C}_{25} \mathrm{H}_{34} \mathrm{O}_{7} \mathrm{Na}$ : 469.2197; found 496.2197. 
Methyl 12S-acetoxy-15,16-epoxy-2-oxo-ent-halima-5(10),13(16),14-trien-18-oate (24) and methyl 12R-acetoxy-15,16-epoxy-2-oxo-ent-halima-5(10),13(16),14-trien-18-oate (25)

To a solution of acetate $22(118 \mathrm{mg}, 0.27 \mathrm{mmol})$ in EtOH $(2.6 \mathrm{~mL})$, aq $\mathrm{HCl}$. (2M, $3.6 \mathrm{~mL})$ was added. The reaction mixture was stirred for $3 \mathrm{~h}$ at room temperaure. Then it was diluted and extracted with $\mathrm{Et}_{2} \mathrm{O}$, washed with water and dried over $\mathrm{Na}_{2} \mathrm{SO}_{4}$. Evaporation of the organic layer yielded 24 (102 mg, 96\%) as a colourless oil; $[\alpha]_{\mathbf{D}}^{22}-5.0$ (c 0.9, $\left.\mathrm{CHCl}_{3}\right)$; IR (film) $v\left(\mathrm{~cm}^{-1}\right)$ 2922, 1734, 1717, 1458, 1374, 1234, 1023; ${ }^{1} \mathrm{H}-\mathrm{NMR}$ (200 MHz): 7.39 (1H, s, H-16), 7.31 (1H, s, H-15), 6.37 (1H, s, H-14), 5.52 (1H, dd, $J=7.0,3.4 \mathrm{~Hz}, \mathrm{H}-12), 3.76$ (3H, s, -COOMe), 2.82-2.63 (2H, m), 2.23-2.06 (2H, m), 2.05-1.50 (5H, m), 1.96 (3H, s, -OCOMe), 1.40-1.20 (3H, m), 1.36 (3H, s, Me-19), 0.95 (3H, d, J = 6.2 Hz, Me-17), 0.84 (3H, s, Me-20); ${ }^{13} \mathrm{C}-\mathrm{NMR}$ (50 MHz): 208.9 (C-2), 174.9 (C-18), 170.2 (-OCOMe), 143.6 (C-16), 140.4 (C-15), 134.3 (C-13), 134.0 (C-10), 125.6 (C-5), 108.8 (C-14), 66.0 (C-12), 52.8 (-COOMe), 49.2 (C-1), 48.8 (C-4), 41.3 (C-11), 41.3 (C-9), 39.8 (C-3), 33.9 (C-8), 26.7 (C-7), 26.0 (C-6), 22.2 (-OCOMe), 21.5 (C-19), 20.7 (C-20), 16.0 (C-17); HRMS (EI) m/z calcd. for $\mathrm{C}_{23} \mathrm{H}_{30} \mathrm{O}_{6} \mathrm{Na}$ : 425.1935; found 425.1944.

Similarly, to a solution of acetate 23 (36 mg, $0.08 \mathrm{mmol})$ in EtOH (0.8 mL), aq HCl. (2M, $1.0 \mathrm{~mL})$ was added. The reaction mixture was stirred for $3 \mathrm{~h}$ at room temperature. Then it was diluted with $\mathrm{Et}_{2} \mathrm{O}$, extracted with $\mathrm{Et}_{2} \mathrm{O}$, washed with water and dried over $\mathrm{Na}_{2} \mathrm{SO}_{4}$. Evaporation of the organic layer yielded 25 (31 mg, 96\%) as a colourless oil; $[\alpha]_{\mathbf{D}}^{22}-21.1$ (c 0.6, $\mathrm{CHCl}_{3}$ ); IR (film) $v\left(\mathrm{~cm}^{-1}\right.$ ) 2922, 1734, 1718, 1458, 1374, 1234, 1119, 1023; ${ }^{1} \mathrm{H}-\mathrm{NMR}$ (200 MHz): 7.39 (1H, s, H-16), 7.36 (1H, s, H-15), 6.37 (1H, s, H-14), 5.83 (1H, dd, $J=8.4,4.8 \mathrm{~Hz}, \mathrm{H}-12$ ), 3.72 (3H, s, -COOMe), 3.27 (1H, d, $J=21.0$ Hz, $\left.\mathrm{H}_{\mathrm{A}}-1\right), 2.99\left(1 \mathrm{H}, \mathrm{d}, J=14.2 \mathrm{~Hz}, \mathrm{H}_{\mathrm{A}}-3\right), 2.83\left(1 \mathrm{H}, \mathrm{d}, J=20.8 \mathrm{~Hz}, \mathrm{H}_{\mathrm{B}}-1\right), 2.31(1 \mathrm{H}, \mathrm{d}, J=14.2 \mathrm{~Hz}$, $\mathrm{H}_{\mathrm{B}}-3$ ), 2.20-1.60 (4H, m), 2.01 (3H, s, -OCOMe), 1.50-1.20 (3H, m), 1.24 (3H, s, Me-19), 0.85 (3H, s, Me-20), 0.83 (3H, d, $J=6.2 \mathrm{~Hz}, \mathrm{Me}-17) ;{ }^{13} \mathrm{C}-\mathrm{NMR}$ (50 MHz): 209.1 (C-2), 175.1 (C-18), 170.4 (OCOOMe), 143.6 (C-16), 140.4 (C-15), 134.0 (C-13), 132.4 (C-10), 126.2 (C-5), 108.9 (C-14), 65.4 (C12), 52.6 (-COOMe), 50.5 (C-1), 49.2 (C-4), 40.4(C-9), 40.1 (C-11), 33.3 (C-8), 29.9 (C-3), 26.8 (C7), 25.7 (C-6), 22.5 (-OCOMe), 22.5 (C-19), 21.8 (C-20), 16.1 (C-17); HRMS (EI) m/z calcd. for $\mathrm{C}_{23} \mathrm{H}_{30} \mathrm{O}_{6} \mathrm{Na}$ : 425.1935; found 425.1945.

Methyl 15,16-epoxy-12S-hydroxy-2-oxo-ent-halima-5(10),13(16),14-trien-18-oate (26)

To a solution of 24 (50 mg, $0.12 \mathrm{mmol}$ ) in methanol (1.0 ml) $\mathrm{Na}_{2} \mathrm{CO}_{3}$ (23 $\mathrm{mg}, 0.21 \mathrm{mmol}$ ) was added. The mixture was stirred at room temperature. After $2 \mathrm{~h}$, the solvent was evaporated and diluted with $\mathrm{Et}_{2} \mathrm{O}$. The organic layer was successively washed with a $2 \mathrm{~N}$ aqueous solution of $\mathrm{HCl}$ and water, dried over $\mathrm{Na}_{2} \mathrm{SO}_{4}$ and evaporated to yield 26 (42 mg, 96\%) as a colourless oil; $[\alpha]_{\mathbf{D}}^{22}-6.1$ (c 1.0, $\mathrm{CHCl}_{3}$ ); IR (film) v (cm $\left.{ }^{-1}\right)$ 3435, 2956, 1724, 1461, 1242, 1120, 1075; ${ }^{1} \mathrm{H}-\mathrm{NMR}$ (200 MHz): 7.34 (2H, m, H-15, H-16), 6.39 (1H, s, H-14), 4.51 (1H, m, H-12), 3.69 (3H, s, -COOMe), 2.78 (1H, d, J = 15.8 $\left.\mathrm{Hz}, \mathrm{H}_{\mathrm{A}}-3\right), 2.71$ (1H, m, $\left.\mathrm{H}_{\mathrm{A}}-1\right), 2.22\left(1 \mathrm{H}, \mathrm{d}, J=15.4 \mathrm{~Hz}, \mathrm{H}_{\mathrm{B}}-3\right)$, 2.19-1.95 (3H, m), 1.93-1.34 (5H, m), 1.25 (3H, s, Me-19), 0.95 (3H, d, $J=6.6 \mathrm{~Hz}, \mathrm{Me}-17$ ), 0-85 (3H, s, Me-20); ${ }^{13} \mathrm{C}-\mathrm{NMR}$ (50 MHz): 207.8 (C-2), 173.9 (C-18), 142.6 (C-16), 137.5 (C-15), 132.8 (C-13), 132.6 (C-10), 129.0 (C-5), 107.2 (C14), 63.1 (C-12), 51.3 (-COOMe), 48.5 (C-1), 47.7 (C-4), 43.1 (C-11), 39.9 (C-9), 38.5 (C-3), 32.7 (C- 
8), 25.5 (C-7), 24.6 (C-6), 21.0 (C-19), 19.8 (C-20), 15.0 (C-17); HRMS (EI) m/z calcd. for $\mathrm{C}_{21} \mathrm{H}_{28} \mathrm{O}_{5} \mathrm{Na}$ : 383.1829; found 383.1829.

Methyl 15,16-epoxy-2R,12S-dihydroxy-ent-halima-5(10),13(16),14-trien-18-oate (27) and 15,16epoxy-12S-hydroxy-ent-halima-5(10),13(16),14-trien-18,2 $\beta$-olide (13)

To an ice cooled solution of 26 (22.0 mg, $0.07 \mathrm{mmol})$ in EtOH $(0.7 \mathrm{~mL}), \mathrm{NaBH}_{4}(13 \mathrm{mg}, 0.33$ $\mathrm{mmol}$ ) was added. After being stirred at room temperature for $3 \mathrm{~h}$, the reaction mixture was recooled to $0^{\circ} \mathrm{C}$ and quenched with a few drops of $2 \mathrm{M}$ aqueous $\mathrm{HCl}$ solution, diluted with EtOAc and water and extracted with EtOAc. The organic layer was washed with water. Evaporation of the dried extract gave a residue, which was chromatographed on silica gel (hex/EtOAc 9/1) to afford 13 (8 mg, 38\%), and 27 (9 mg, 43\%). Compound 27: a colourless oil; $[\alpha]_{\mathbf{D}}^{22}-41.1$ (c 0.6, $\mathrm{CHCl}_{3}$ ); IR (film) $v\left(\mathrm{~cm}^{-1}\right.$ ) 3408, 2929, 1727, 1460, 1274, 1161, 1024; ${ }^{1} \mathrm{H}-\mathrm{NMR}$ (200 MHz): 7.20 (2H, m, H-15, H-14), 6.41 (1H, bs, H-14), 3.97 (2H, m, H-2, H-12), 3.66 (3H, s, -COOMe), 2.42- 1.95 (5H, m), 1.92-1.47 (6H, m), 1.33 (3H, s, Me-19), 0.93 (3H, s, Me-20), 0.85 (3H, d, $J=6.6 \mathrm{~Hz}, \mathrm{Me}-17) ;{ }^{13} \mathrm{C}-\mathrm{NMR}$ (50 MHz): 177.9 (C-18), 143.4 (C-16), 138.8(C-15), 133.9 (C-10), 131.4 (C-5), 130.8 (C-13), 108.8 (C-14), 65.5 (C-2), 64.9 (C12), 52.6 (-COOMe), 49.2 (C-4), 47.3 (C-3), 45.0 (C-1), 40.6 (C-9), 35.4 (C-8), 29.3 (C-7), 26.5 (C-6), 22.1 (C-20), 16.1(C-19), 14.3 (C-17); HRMS (EI) m/z calcd. for $\mathrm{C}_{21} \mathrm{H}_{30} \mathrm{O}_{5}$ Na: 385.1985; found 385.1969. Compound 13: see above.

\section{5,16-Epoxy-12-oxo-ent-halima-5(10),13(16),14-trien-18,2 $\beta$-olide (2)}

To a mixture of 13 (3 mg, $0.01 \mathrm{mmol}$ ) $N$-methylmorpholine- $N$-oxide (NMO) (4 mg, $0.03 \mathrm{mmol}$ ) and molecular sieves (15 mg) in anhydrous $\mathrm{CH}_{2} \mathrm{Cl}_{2}(0.3 \mathrm{~mL})$ under an $\mathrm{Ar}$ atmosphere and at room temperature, TPAP (3.0 mg, $3 \times 10^{-3} \mathrm{mmol}$ ) was added. The reaction mixture was stirred for $30 \mathrm{~min}$. and then filtered on silica gel and Celite (DCM and EtOAc). Evaporation of the solvent yielded 2 (3.0 mg, 92\%).

\section{Acknowledgements}

The authors are grateful to Dr. A. M. Lithgow and Dr. C. Raposo, for the NMR and mass spectra respectively. The authors also thank the CICYT (CTQ2005-04406) and FSE for financial support and to Junta de Castilla y León for a doctoral fellowship to A.C.A.

\section{References}

1. Evans, F. J.; Taylor, S. E. Pro-Inflammatory. Tumour-Promoting and Anti-Tumour Diterpenes of the Plant Families Euphorbiaceae and Thymelaeaceae. Fortschr. Chem. Org. 1983, 44, 1-90

2. Siems, K.; Jakupovic, J.; Castro, V.; Poveda, L. Constituents of two Acalypha Species. Phytochemistry 1996, 41, 851-853.

3. Hecker, E.; Schmidt, R. Phorbolesters- the Irritants and Cocarcinogens of Croton Tigilium L. Fortschr. Chem. Org. 1974, 31, 377-458. 
4. Marcos, I. S.; Hernández, F. A.; Sexmero, M. J.; Díez, D.; Basabe, P.; Pedrero, A. B.; Garcia, N.; Urones, J. G. Synthesis and absolute configuration of (-)-chettaphanin I and (-)-chettaphanin II. Tetrahedron 2003, 59, 685-694.

5. Sato, A.; Kurabayashi, M.; Nagahori, H.; Ogiso, A.; Mishima, H.; Chettaphanin I, a novel furano diterpenoid. Tetrahedron Lett. 1970, 11, 1095-1098.

6. Marcos, I. S.; Hernández, F. A.; Sexmero, M. J.; Díez, D.; Basabe, P.; Pedrero, A. B.; García, N.; Sanz, F.; Urones, J. G. Synthesis and absolute configuration of (-)-chettaphanin II. Tetrahedron Lett. 2002, 43, 1243-1245.

7. Sato, A.; Kurabayashi, M.; Ogiso, A.; Mishima, H.; Chettaphanin II, a novel furano diterpenoid. Tetrahedron Lett. 1971, 12, 839-842.

8. Kanlayavattanakul, M.; Ruangrungsi, N.; Watanabe, T.; Kawahata, M.; Therrien, B.; Yamaguchi, K.; Ishikawa, T. ent-Halimane Diterpenes and a Guaiane Sesquiterpene from Cladogynos orientalis J. Nat. Prod. 2005, 68, 7-10.

9. Ley, S. V.; Norman, J.; Griffith, W. P.; Marsden, S. P. Tetrapropylammonium Perrutenate $\mathrm{Pr}_{4} \mathrm{~N}^{+} \mathrm{RuO}_{4}^{-}$, TPAP: A Catalytic Oxidant for Organic Synthesis. Synthesis 1994, 639-666.

10. Zoretic, P. A.; Fang, H.; Ribeiro, A. A.; Dubay, G. Synthesis of Acuminolide from (+)-Sclareolide. J. Org. Chem. 1998, 63, 1156-1161.

11. Magnuson, S. R.; Sepp-Lorenzino, L.; Rosen, N.; Danishefsky, S. J. A Concise Total Synthesis of Dysidiolide through Application of a Dioxolenium-Mediated Diels-Alder Reaction. J. Am. Chem. Soc. 1998, 120, 1615-1616.

12. Boukouvalas, J.; Wang, J. X.; Marion, O.; Ndzi, B. Synthesis and Stereochemistry of the Antitumor Diterpenoid (+)-Zerumin B J. Org. Chem. 2006, 71, 6670-6673.

13. Marcos, I. S.; Pedrero, A. B.; Sexmero, M. J.; Díez, D.; Basabe, P.; García, N.; Moro, R. F.; Broughton, H. B.; Mollinedo, F.; Urones. J. G. Synthesis of Bioactive Sesterterpenolides from entHalimic Acid. 15-Epi-ent-cladocoran A and B. J. Org. Chem. 2003, 68, 7496-7504.

14. Marcos, I. S.; Escola, M. A.; Moro, R. F.; Basabe, P.; Díez, D.; Sanz, F.; Mollinedo, F.; de la Iglesia-Vicente, J.; Sierra B. G.; Urones, J. G. Synthesis of novel antitumoural analogues of dysidiolide fron ent-halimic acid. Bioorg. Med. Chem. 2007, 15, 5719-5737.

15. Maryanoff, B. E.; Reitz, A. B. The Wittig Olefination Reaction and Modifications Involving Phosphoryl-Stabilized Carbanions. Stereochemistry, Mechanism, and Selected Synthetic Aspects. Chem. Rev. 1989, 89, 863-927.

16. Vedejs, E.; Fleck, T. J. Kinetic (Not equilibrium) Factors are dominating in Wittig Reactions of Conjugated Ylides. J. Am. Chem. Soc. 1989, 111, 5861-5871.

17. Kelly, S. E. Alkene Synthesis. Phosphorus-Stabilized Alkenation; In Comprehensive Organic Synthesis; Trost, B. M.; Fleming, I., Eds. Pergamon Press: Oxford, 1991; Vol. 1, pp. 755-782.

18. Schröder, M. Osmium Tetraoxide Cis Hydroxilation of Unsaturated Substrates Chem. Rev. 1980, 80, 187-213.

19. Lohray, B. B. Recent advances in the asymmetric dihydroxilation of alkenes. Tetrahedron Asymm. 1992, 3, 1317-1349.

20. Haines, A. H. Reactions with Formation of Carbon-Oxygen Bonds: (iii) Glycol Forming Reactions; In Comprehensive Organic Synthesis; Trost, B. M.; Fleming, I., Eds. Pergamon Press: Oxford, 1991; Vol. 7, pp. 437-448. 
21. Oliver, S. F.; Högenauer, K.; Simic, O.; Antonello, A.; Smith, M. D.; Ley, S. V. A route to the Thapsigargins from (S)-Carvone Providing a Substrate-Controlled Total Synthesis of Tribolide, Nortribolide and Thapsivillosin F. Angew. Chem. Int. Ed. Engl. 2003, 42, 5996-6000.

Sample Availability: Samples of compounds $\mathbf{1}$ and $\mathbf{6}$ are available from the authors. Copies of the spectra for all compounds are also available on request.

(C) 2008 by the authors; licensee Molecular Diversity Preservation International, Basel, Switzerland. This article is an open-access article distributed under the terms and conditions of the Creative Commons Attribution license (http://creativecommons.org/licenses/by/3.0/). 